\title{
Muon spin relaxation and fluctuating magnetism in the pseudogap phase of YBa2 Cu3 Oy
}

\author{
Z. H. Zhu, J. Zhang, Z. F. Ding, C. Tan, C. S. Chen, Q. Wu, Y. X. \\ Yang, O. O. Bernal, P.-C. Ho, G. D. Morris, A. Koda, A. D. Hillier, S. \\ P. Cottrell, P. J. Baker, P. K. Biswas, J. Qian, X. Yao, D. E. \\ MacLaughlin, and L. Shu
}

\section{Published version information}

Citation: ZH Zhu et al. Muon spin relaxation and fluctuating magnetism in the pseudogap phase of YBa2 Cu3 Oy. Phys Rev B 103, no. 13 (2021): 134426

DOI: $10.1103 /$ PhysRevB.103.134426

This version is made available in accordance with publisher policies. Please cite only the published version using the reference above. This is the citation assigned by the publisher at the time of issuing the APV. Please check the publisher's website for any updates. 


\title{
Muon spin relaxation and fluctuating magnetism in the pseudogap phase of $\mathrm{YBa}_{2} \mathrm{Cu}_{3} \mathrm{O}_{y}$
}

\author{
Z. H. Zhu, ${ }^{1, *}$ J. Zhang, ${ }^{1,}{ }^{*}$ Z. F. Ding, ${ }^{1}$ C. Tan, ${ }^{1}$ C. S. Chen $\odot,{ }^{1}$ Q. Wu, ${ }^{1}$ Y. X. Yang, ${ }^{1}$ O. O. Bernal $\odot,{ }^{2}$ P.-C. Ho, ${ }^{3}$ G. D. Morris, ${ }^{4}$ \\ A. Koda, ${ }^{5,6}$ A. D. Hillier, ${ }^{7}$ S. P. Cottrell, ${ }^{7}$ P. J. Baker, ${ }^{7}$ P. K. Biswas, ${ }^{7}$ J. Qian, ${ }^{8}$ X. Yao, ${ }^{8,9}$ D. E. MacLaughlin, ${ }^{10}$ \\ and L. Shu (10) $1,9,11, \dagger$ \\ ${ }^{1}$ State Key Laboratory of Surface Physics, Department of Physics, Fudan University, Shanghai 200433, People's Republic of China \\ ${ }^{2}$ Department of Physics and Astronomy, California State University, Los Angeles, California 90032, USA \\ ${ }^{3}$ Department of Physics, California State University, Fresno, California 93740, USA \\ ${ }^{4}$ TRIUMF, Vancouver, British Columbia, Canada V6T $2 A 3$ \\ ${ }^{5}$ Muon Science Laboratory and Condensed Matter Research Center, Institute of Materials Structure Science, \\ High Energy Accelerator Research Organization (KEK), Tsukuba, Ibaraki 305-0801, Japan \\ ${ }^{6}$ Department of Materials Structure Science, The Graduate University for Advanced Studies (Sokendai), Tsukuba, Ibaraki 305-0801, Japan \\ ${ }^{7}$ ISIS Facility, STFC Rutherford Appleton Laboratory, Harwell Science and Innovation Campus, Chilton, Didcot, \\ Oxon OX11 0QX, United Kingdom \\ ${ }^{8}$ Key Laboratory of Artificial Structures and Quantum Control (Ministry of Education), School of Physics and Astronomy, \\ Shanghai Jiao Tong University, Shanghai 200240, People's Republic of China \\ ${ }^{9}$ Collaborative Innovation Center of Advanced Microstructures, Nanjing 210093, People's Republic of China \\ ${ }^{10}$ Department of Physics and Astronomy, University of California, Riverside, Riverside, California 92521, USA \\ ${ }^{11}$ Shanghai Research Center for Quantum Sciences, Shanghai 201315, People's Republic of China
}

(Received 10 August 2020; revised 29 March 2021; accepted 5 April 2021; published 19 April 2021)

\begin{abstract}
We report the results of a muon spin relaxation study of slow magnetic fluctuations in the pseudogap phase of underdoped single-crystalline $\mathrm{YBa}_{2} \mathrm{Cu}_{3} \mathrm{O}_{y}, y=6.77$ and 6.83. The dependence of the dynamic muon spin relaxation rate on applied magnetic field yields the rms magnitude $B_{\mathrm{loc}}^{\mathrm{rms}}$ and correlation time $\tau_{c}$ of fluctuating local fields at muon sites. The observed relaxation rates do not decrease with decreasing temperature below the pseudogap onset at $T^{*}$, as would be expected for a conventional magnetic transition; both $B_{\mathrm{loc}}^{\mathrm{rms}}$ and $\tau_{c}$ are roughly constant between $T^{*}$ and the superconducting transition. NMR relaxation rates due to putative loop-current fluctuations are estimated and found to be too small to be observed. Our results put strong constraints on the theories of the anomalous pseudogap magnetism in $\mathrm{YBa}_{2} \mathrm{Cu}_{3} \mathrm{O}_{y}$.
\end{abstract}

DOI: 10.1103/PhysRevB.103.134426

\section{INTRODUCTION}

The pseudogap phase in hole-doped cuprates is one of the most studied quantum states in high-temperature superconductors [1-10]. It is characterized by the loss of low-lying electronic excitations, and it emerges below a characteristic temperature $T^{*}$ that depends strongly on the hole concentration on the $\mathrm{CuO}_{2}$ plane. Anomalous transport [11], thermodynamic [11,12], and electrodynamic [6] properties are observed below $T^{*}$. Extensive work [2] has shown that the pseudogap state is quite different from a normal metal, and it is widely believed that it holds the key to a general model for high-temperature superconductivity. Two categories of theories, involving either a crossover $[1,3,10]$ or a true thermodynamic phase transition with a quantum critical point [4,8], attempt to explain the origin of the pseudogap. Both are consistent with several experimental phenomena [10].

\footnotetext{
${ }^{*}$ These authors contributed equally to this work.

${ }^{\dagger}$ Author to whom all correspondence should be addressed: leishu@fudan.edu.cn
}

A variety of symmetry-sensitive techniques have discovered broken inversion and time-reversal symmetries (TRS) in a number of cuprate superconductors below $T^{*}$ [13-19]. Among the various models, theories that posit intra-unitcell (IUC) magnetic order [20-23] have been proposed in which both of these symmetries are broken. Polarized neutron diffraction (PND) experiments yielded evidence for TRSbreaking ordered IUC moments below $T^{*}$ of the order of $0.1 \mu_{\mathrm{B}}[13,14,16,24]$, but also for the absence of such moments [25,26]; the latter has, however, been refuted [27,28]. Resonant inelastic x-ray experiments [29] observe magnetic correlations across a wide family of cuprates from under- to overdoping, albeit at low temperature.

Objections to IUC magnetism were raised, however, as probes of magnetic moments and local fields expected from TRS breaking yielded a wide variety of results. Claims of both the presence [30-32] and absence [33-38] of static and/or dynamic fields in the pseudogap phase have been made based on muon spin relaxation ( $\mu \mathrm{SR}$ ) experiments [39-41] carried out using different configurations. NMR experiments [42-44] have not observed such fields. The current consensus is that there is no evidence for static magnetic order from either $\mu \mathrm{SR}$ or NMR. However, most $\mu \mathrm{SR}$ data were taken in zero 
field (ZF) [30,32-38,45], where static and dynamic fields both contribute to the relaxation [39-41]. In longitudinal-field (LF) $\mu \mathrm{SR}$ experiments, relaxation in a sufficiently strong field is solely dynamic and is therefore a probe of fluctuating magnetism.

Only a small number of LF- $\mu$ SR measurements in the pseudogap phase have been reported [31,32,37,38], most with very few data in strong LF. Dynamic muon spin relaxation by slowly fluctuating magnetic fields was, however, observed in recent LF- $\mu$ SR experiments in $\mathrm{YBa}_{2} \mathrm{Cu}_{3} \mathrm{O}_{y}$ [31]. Measured relaxation rates $\lambda$ in the pseudogap phase yielded heuristic estimates of $\sim 100 \mathrm{mT}$ for the rms fluctuating local field $B_{\text {loc }}^{\text {rms }}$ at muon sites, and $\sim 10^{-8} \mathrm{~s}$ for the correlation time $\tau_{c}$ of the fluctuations. The fluctuations are consistent with "static" IUC order from PND experiments, for which the experimental timescale $\left(\sim 10^{-12} \mathrm{~s}\right)$ is much shorter than $\tau_{c}$. The fluctuations also explain the absence of static fields in NMR and $\mu$ SR experiments, where timescales for quasistatic behavior are considerably longer $\left(\gtrsim 10^{-5} \mathrm{~s}\right)$.

Maxima in $\lambda(T)$ were observed at temperatures $T_{\mathrm{mag}} \approx T^{*}$ in $\mathrm{YBa}_{2} \mathrm{Cu}_{3} \mathrm{O}_{y}, y=6.72,6.77$, and 6.95 , followed by increases of $\lambda$ with decreasing temperature in the pseudogap phase [31]. It was determined that the maxima were not due to activated muon hopping, charge inhomogeneity, nucleardipole fields, or other phenomena. In addition, the values of $B_{\mathrm{loc}}^{\mathrm{rms}}$ were consistent with the IUC moments observed in PND experiments. These results are strong evidence for fluctuating IUC magnetic order in the pseudogap phase. A recent $\mu \mathrm{SR}$ study of $\mathrm{Bi}_{2+x} \mathrm{Sr}_{2-x} \mathrm{CaCu}_{2} \mathrm{O}_{8+\delta}$ [32] found quasistatic magnetic fluctuations that might have the same origin as those in $\mathrm{YBa}_{2} \mathrm{Cu}_{3} \mathrm{O}_{y}$. Comparison between different cuprate materials cannot be made due to the dearth of LF- $\mu$ SR results.

In this article we report the temperature dependencies of $B_{\mathrm{loc}}^{\mathrm{rms}}$ and $\tau_{c}$ in the pseudogap phase of $\mathrm{YBa}_{2} \mathrm{Cu}_{3} \mathrm{O}_{y}, y=6.77$ and 6.83 , from LF- $\mu$ SR measurements of dynamic relaxation rates $\lambda_{L}$. The oxygen concentrations were chosen so that $T^{*}$ is above the onsets of charge density wave phases $[10,46]$ but well below the muon hopping temperature regime $T \gtrsim 200 \mathrm{~K}$ [31]. We determine $B_{\mathrm{loc}}^{\mathrm{rms}}$ and $\tau_{c}$ separately by extracting them from the field dependence of $\lambda_{L}$ measured at a number of temperatures. We find that they are both roughly constant in the pseudogap phase down to the superconducting transition temperature $T_{c}$. This is in contrast to ordinary magnetic transitions, where both quantities decrease with decreasing temperature in the ordered state [47], and it raises the question of the origin of the spin dynamics at low temperatures. It has been suggested [48] that the fluctuations arise from quantum size effects in domains of IUC order.

\section{EXPERIMENT}

Parent compounds for the single crystalline samples of $\mathrm{YBa}_{2} \mathrm{Cu}_{3} \mathrm{O}_{y}, y=6.77$ and 6.83 , were synthesized by a polythermal top-seeded solution-growth method using a $3 \mathrm{BaO}-5 \mathrm{CuO}$ solvent flux [49]. This method can yield crystals with high crystallinity [50]. The bulk single crystal was then cut into small pieces with $a b$ plane lateral dimensions of $2 \mathrm{~mm} \times 2 \mathrm{~mm}$ and $c$-axis thicknesses of $0.5 \mathrm{~mm}$. Oxygen concentrations $y=6.77$ and 6.83 were achieved by postannealing the parent compound in flowing ultrahigh-purity oxygen at
TABLE I. Doping level $p$, superconducting transition temperatures $T_{c}$, pseudogap onset temperatures $T^{*}$, and peak temperatures $T_{\text {mag }}$ in $\mu^{+}$relaxation rates for $\mathrm{YBa}_{2} \mathrm{Cu}_{3} \mathrm{O}_{y}, y=6.77$ and 6.83. From Ref. [31] except as noted.

\begin{tabular}{lcccc}
\hline \hline$y$ & $p$ & $T_{c}(\mathrm{~K})$ (onset) & $T^{*}(\mathrm{~K})$ (approx.) & $T_{\operatorname{mag}}(\mathrm{K})$ \\
\hline 6.77 & 0.138 & 80 & $155-185$ & $160(10)$ \\
6.83 & 0.149 & 88 & $130-160$ & $142(10)^{\mathrm{a}}$ \\
\hline \hline
\end{tabular}

${ }^{\mathrm{a}}$ Unpublished data.

different temperatures [31,51,52]. Values of $y$ and doping level $p$ were obtained from measurements of $T_{c}$. $T^{*}$ was determined as the temperature for which the resistivity departs from $T$-linear behavior at high temperatures (Fig. $4 \mathrm{C}$ of Ref. [31]). For comparison, values of $T_{c}, T^{*}$, and $T_{\text {mag }}$ are given in Table I.

$\mu$ SR experiments were carried out using the LAMPF spectrometer at TRIUMF, Vancouver, Canada; the ARTEMIS spectrometer at J-PARC, Tokai, Japan; and the EMU spectrometer at the ISIS Facility, Rutherford Appleton Laboratory, Chilton, UK. At all facilities, $100 \%$ spin-polarized positively charged muons $\left(\mu^{+}\right)$were implanted into the samples with the initial $\mu^{+}$spin polarization $\mathbf{P}_{\mu}(0)$ normal to the $a b$ plane. Appropriate functional forms were least-squares fit to the asymmetry data using the MUSRFIT $\mu \mathrm{SR}$ analysis program [53].

Previous $\mu$ SR experiments on $\mathrm{YBa}_{2} \mathrm{Cu}_{3} \mathrm{O}_{y}$ [31] revealed that fluctuations of local fields at $\mu^{+}$sites are motionally narrowed: $\gamma_{\mu} B_{\mathrm{loc}}^{\mathrm{rms}} \tau_{c} \ll 1$, where $\gamma_{\mu}=8.5156 \times 10^{8} \mathrm{~s}^{-1} \mathrm{~T}^{-1}$ is the muon gyromagnetic ratio. In an externally applied longitudinal field $\mathbf{H}_{L} \| \mathbf{P}_{\mu}(0)$, the corresponding motionally narrowed relaxation rate $\lambda_{L}$ follows the so-called Redfield relation $[54,55]$

$$
\lambda_{L}\left(H_{L}\right)=\frac{2\left(\gamma_{\mu} B_{\mathrm{loc}}^{\mathrm{rms}}\right)^{2} \tau_{c}}{1+\left(\gamma_{\mu} \mu_{0} H_{L} \tau_{c}\right)^{2}}
$$

if the fluctuations are Markovian and characterized by a single correlation time $\tau_{c}$. Equation (1) represents the effect of sweeping the $\mu^{+}$Zeeman frequency $\gamma_{\mu} \mu_{0} H_{L}$ through the fluctuation noise spectrum, and it assumes no field dependence of the spin dynamics. A crossover occurs for $\gamma_{\mu} \mu_{0} H_{L} \approx 1 / \tau_{c}$, and the area $\int_{0}^{\infty} \lambda_{L}\left(H_{L}\right) d H_{L}=\pi \gamma_{\mu} B_{\mathrm{loc}}^{2}$ is independent of $\tau_{c}$. For more general fluctuation spectra, $\lambda_{L}\left(H_{L}\right)$ decreases with increasing $H_{L}$ as the $\mu^{+}$Zeeman frequency passes through a high-frequency cutoff. The Redfield relation has been widely applied in $\mu$ SR to characterize dynamic fluctuating magnetic fields in strongly correlated electron systems [56-58].

We measured $\lambda_{L}\left(H_{L}\right)$ in $\mathrm{YBa}_{2} \mathrm{Cu}_{3} \mathrm{O}_{y}, y=6.77$ and 6.83, at various temperatures above the superconducting transitions. All data were taken using the LAMPF spectrometer except for $y=6.83, T=170 \mathrm{~K}$, for which the ARTEMIS spectrometer was used.

Figure 1(a) shows representative asymmetry versus time spectra from $\mathrm{YBa}_{2} \mathrm{Cu}_{3} \mathrm{O}_{6.77}, T=95 \mathrm{~K}, \mu_{0} H_{L}=6$ and $280 \mathrm{mT}$. The decrease in relaxation rate with increasing $\mathrm{LF}$ expected from Eq. (1) is small but resolved. The absence of deviation in the residuals and the good statistical $\chi^{2}$ values 


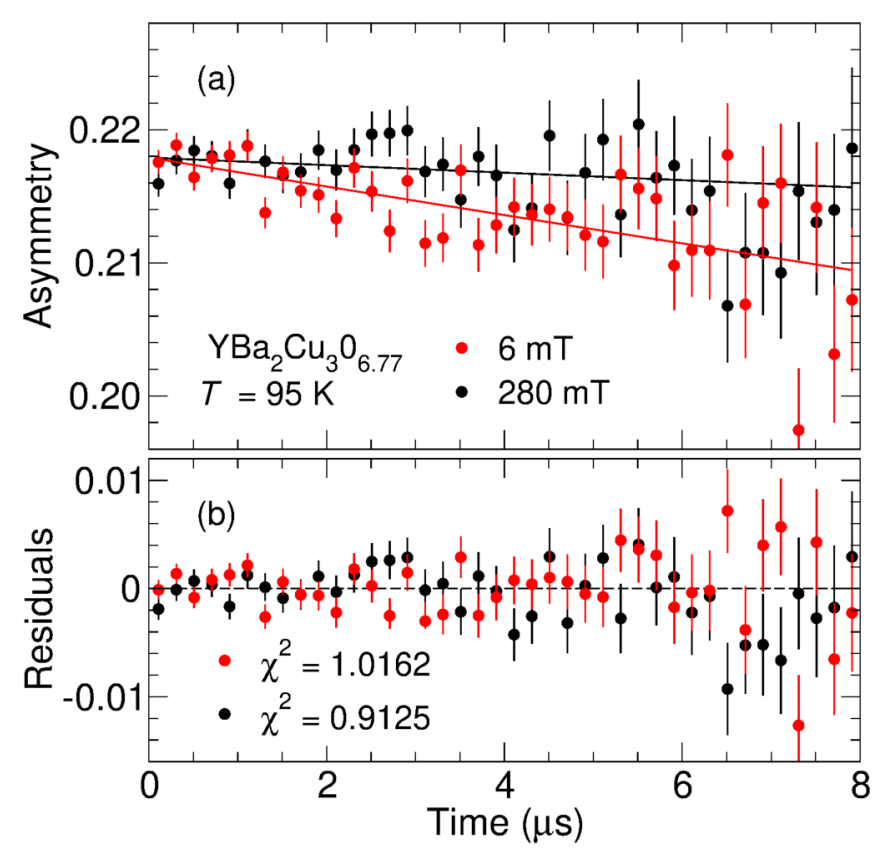

FIG. 1. (a) Representative LF- $\mu$ SR asymmetry time spectra from $\mathrm{YBa}_{2} \mathrm{Cu}_{3} \mathrm{O}_{6.77}, T=95 \mathrm{~K}, \mu_{0} H_{L}=6$ and $280 \mathrm{mT}$. Curves: fits of exponential relaxation functions (linear for slow relaxation). Uncertainties are statistical and one standard deviation. (b) Residuals (data - fits) and reduced $\chi^{2}$ values.

[Fig. 1(b)] show that the data are well characterized by the fits.

The observed $\mu^{+}$spin relaxation in $\mathrm{YBa}_{2} \mathrm{Cu}_{3} \mathrm{O}_{y}$ is very slow [31], and care must be taken to characterize spurious spectrometer-dependent signals. $\mu^{+}$relaxation is even slower in pure silver [31,59], so that control experiments on $\mathrm{Ag}$ samples serve as a check for such signals. LF- $\mu$ SR data were taken on the LAMPF and ARTEMIS spectrometers using a pure silver sample with lateral dimension and thickness similar to those of the $\mathrm{YBa}_{2} \mathrm{Cu}_{3} \mathrm{O}_{y}$ samples. Figure 2 shows the field dependencies of $\mu^{+}$dynamic relaxation rates $\lambda_{\mathrm{Ag}}$ measured on the spectrometers used in this study. Data from the EMU spectrometer, taken from the supplementary material for Ref. [31], are also shown. The values $\lambda_{\mathrm{Ag}} \approx 1 \mathrm{~ms}^{-1}$ are in good agreement with previous results [59], and they serve as a field-independent upper bound on any such signal up to $400 \mathrm{mT}$. They are evidence for the absence of strong spectrometer-dependent effects.

Small spectrometer-dependent corrections, different for the LAMPF and ARTEMIS spectrometers, were, however, necessary because of the very slow relaxation rates. In the TRIUMF VG-Quant gas-flow cryostat used in the LAMPF spectrometer, the sample is suspended in a copper frame. Muons that miss the sample "fly past" and are vetoed by downstream counters. However, "empty-frame" data taken with the sample removed revealed that a few percent of the muon flux stopped in the frame and relaxed at a significant rate, resulting in a spurious signal. Use of the empty-frame results to correct the observed LF relaxation data is described in Appendix A.

In the ARTEMIS cryostat, a sizable weakly relaxing contribution to the signal was observed from muons that missed the sample and stopped in the silver sample holder; this is

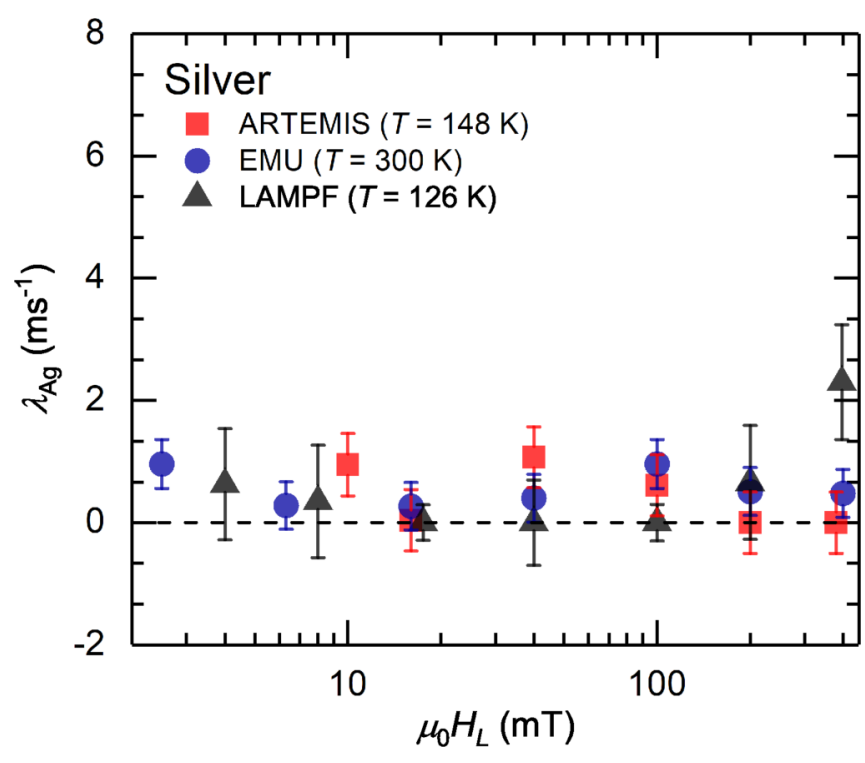

FIG. 2. LF muon spin relaxation rates in pure silver samples with dimensions similar to those of the $\mathrm{YBa}_{2} \mathrm{Cu}_{3} \mathrm{O}_{y}$ samples. Data taken using three different spectrometers at different temperatures. Squares: J-PARC/ARTEMIS. Circles: ISIS/EMU (from Ref. [31]). Triangles: TRIUMF/LAMPF.

typical for many $\mu \mathrm{SR}$ cryostats. Its amplitude was measured using ZF data, where the sample and Ag contributions could be separately determined because of their different relaxation rates. The correction for ARTEMIS LF data, also described in Appendix A, uses Ag rates from Fig. 2.

\section{RESULTS}

\section{A. Field dependence of relaxation rate}

$$
\text { 1. } y=6.77
$$

Figure 3 shows the field dependence of $\lambda_{L}$ for $y=6.77$ at a number of temperatures. Data for $T=85 \mathrm{~K}$ were reported in Ref. [31] and are not shown here. Fits to Eq. (1) are shown for $95 \leqslant T \leqslant 188 \mathrm{~K}$. The latter is higher than $T_{\mathrm{mag}}$, but all

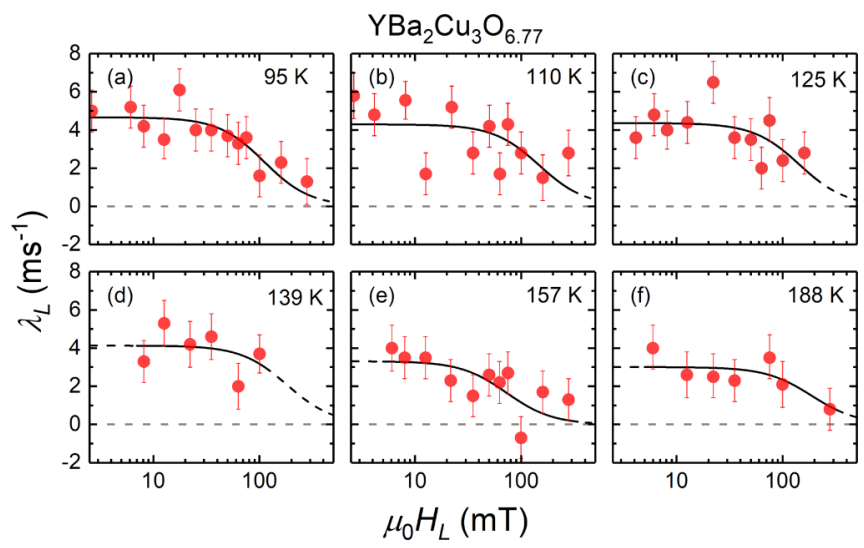

FIG. 3. Dependence of dynamic $\mu^{+}$relaxation rate $\lambda_{L}$ on longitudinal field $H_{L}$ in $\mathrm{YBa}_{2} \mathrm{Cu}_{3} \mathrm{O}_{6.77}, T>T_{c}$. Solid curves: fits of Eq. (1) to the data. Dashed curves: extensions of fits to regions outside data ranges. 


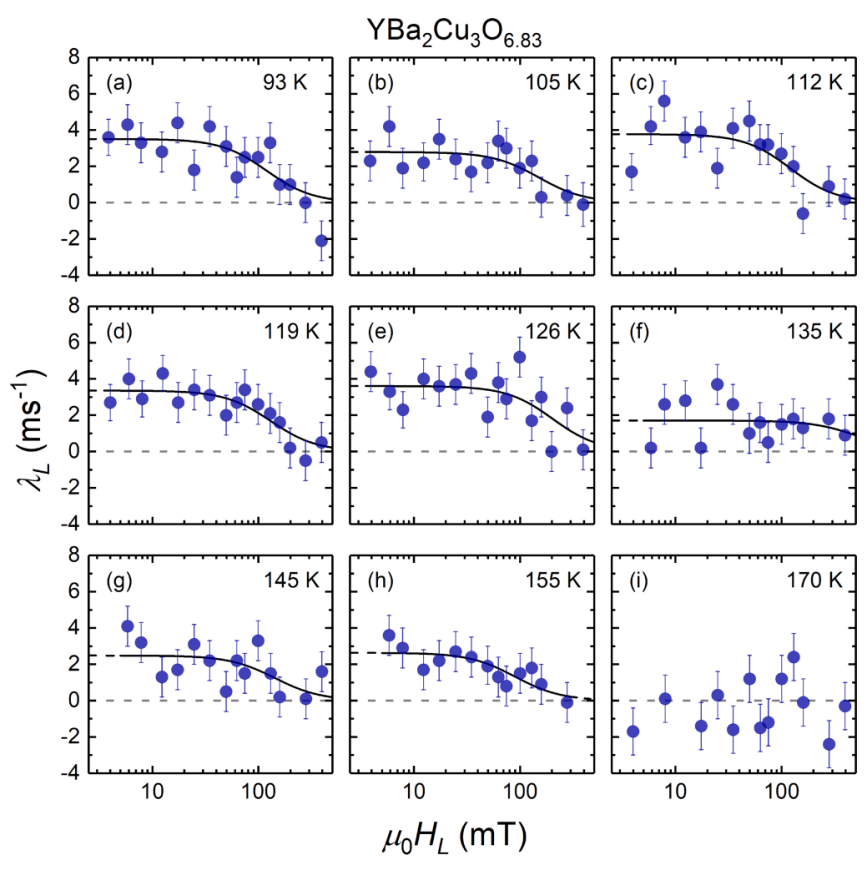

FIG. 4. Dependence of $\lambda_{L}$ on $H_{L}$ in $\mathrm{YBa}_{2} \mathrm{Cu}_{3} \mathrm{O}_{6.83}, T>T_{c}$. Solid and dashed curves as in Fig. 3.

temperatures are in or below the range of reported $T^{*}$ values (Table I). The half-widths of the fit curves, which, as noted above, are measures of $1 / \tau_{c}$, are of the order of $100 \mathrm{mT}$, corresponding to $\tau_{c} \approx 10 \mathrm{~ns}$. At $157 \mathrm{~K}$ and above there is a significant decrease in the relaxation rate at low fields, possibly due to a spatially inhomogeneous distribution of $T^{*}$ in the sample.

$$
\text { 2. } y=6.83
$$

Corresponding results for $y=6.83$ are shown in Fig. 4. A Redfield field dependence of $\lambda_{L}\left(H_{L}\right)$ is again observed for temperatures up to and slightly above $T_{\text {mag }}$, but at $170 \mathrm{~K}$ (above the range of $T^{*}$ ) the rate has fallen to zero to within errors. This is discussed below in Sec. III B.

Statistical data for these fits are discussed in Appendix B.

\section{B. Temperature dependencies}

Figure 5 shows the temperature dependencies of $B_{\text {loc }}^{\text {rms }}$ and $\tau_{c}$ obtained from the fits shown in Figs. 3 and 4 . Values of $B_{\text {loc }}^{\text {rms }}$ agree with previous results [31]. There is a slight but statistically marginal increase in $\tau_{c}$ with decreasing temperature below $T^{*}$ for both dopings, which, however, is accompanied by a decrease in $B_{\text {loc }}^{\text {rms }}$; it can be seen that the two parameters are strongly anticorrelated statistically, a property of Eq. (1). Thus the data are suggestive but not conclusive. Results for $y=6.83, T=93 \mathrm{~K}$ differ from those previously reported [31], most likely due to less uncertainty in the present data.

For $y=6.83, T=170 \mathrm{~K}\left(\gtrsim 10 \mathrm{~K}\right.$ above $\left.T^{*}\right)$, essentially no relaxation was observed within errors up to $\mu_{0} H_{L}^{\max }=0.4 \mathrm{~T}$ [Fig. 4(i)]. This and the consequent absence of a high-field cutoff put an upper bound $1 /\left(\gamma_{\mu} \mu_{0} H_{L}^{\max }\right) \sim 2$ ns on $\tau_{c}$. We note that if fluctuating magnetism exists above $T^{*}$ [60], then

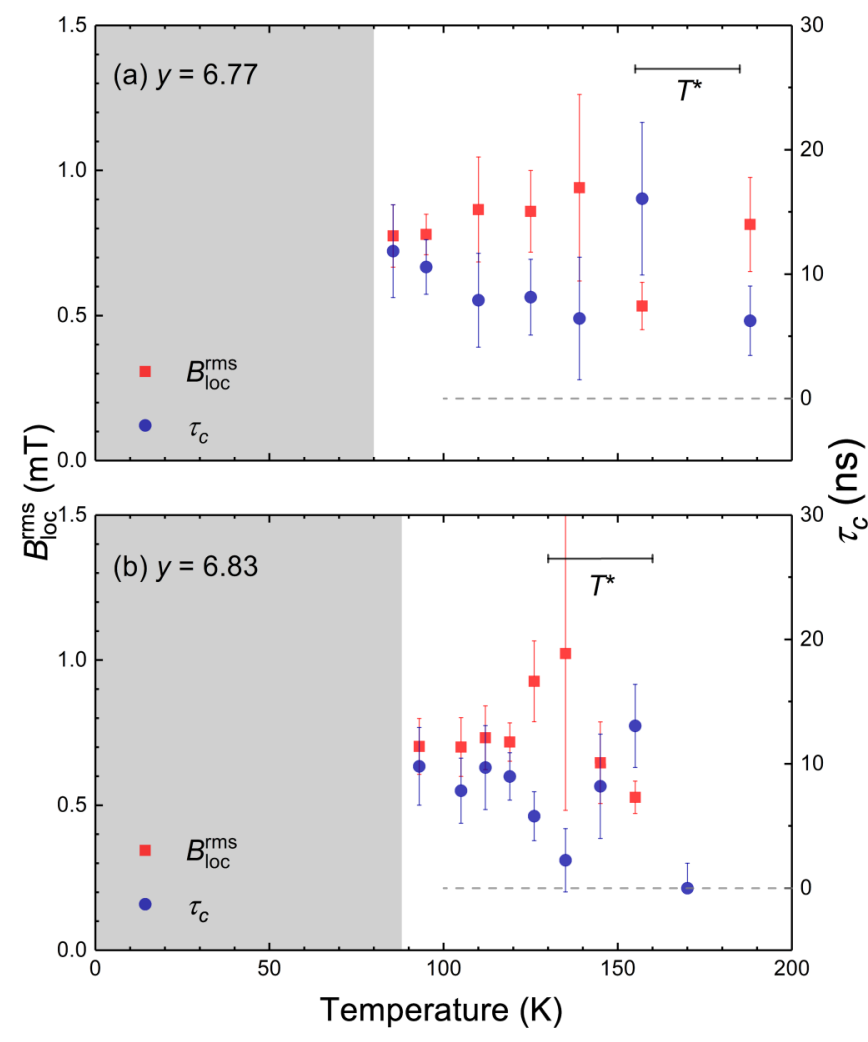

FIG. 5. Temperature dependencies of $\mu^{+}$rms local fields $B_{\text {loc }}^{\mathrm{rms}}$ and correlation times $\tau_{c}$ in $\mathrm{YBa}_{2} \mathrm{Cu}_{3} \mathrm{O}_{y}$. (a) $y=6.77$. (b) $y=6.83$. Shaded areas: superconducting phase. Horizontal bars: ranges of reported pseudogap onset temperatures $T^{*}$.

$B_{\text {loc }}^{\mathrm{rms}}$ is nonzero there and thus is not an order parameter for the pseudogap phase.

Due to time limitations at the accelerator facilities, not enough temperature points could be taken to resolve the previously observed peaks in $\lambda_{L}(T)$ at $T_{\text {mag }} \approx T^{*}$ [31]. As noted above, the LF- $\mu$ SR results in this earlier study were associated with the pseudogap phase by these peaks and by the consistency of $B_{\text {loc }}^{\text {rms }}$ with the IUC moments observed by PND.

\section{DISCUSSION}

\section{A. Fluctuations and PND}

As has been previously noted [31], values of $B_{\mathrm{loc}}^{\mathrm{rms}} \approx$ $1 \mathrm{mT}$ (Fig. 5) are consistent with calculated local fields from $\sim 0.1 \mu_{B}$ IUC magnetic moments observed by PND in $\mathrm{YBa}_{2} \mathrm{Cu}_{3} \mathrm{O}_{y}$ [16]. The fluctuations would not affect the PND results; the consequent broadening $\hbar / \tau_{c}$ of the Bragg reflections is $\sim 0.1 \mu \mathrm{eV}$, much smaller than the PND energy resolution $\sim 1 \mathrm{meV}$ [48].

\section{B. Conflicting conclusions}

As mentioned in the Introduction, a series of $\mu \mathrm{SR}$ studies [30-37] reported conflicting results on the nature of the detected magnetic fields in the pseudogap states of several hole-doped cuprates. Concerns were raised [45] whether the putative IUC field is instead associated with charge inhomogeneity or muon diffusion effects. However, it has been 
TABLE II. Muon and nuclear gyromagnetic ratios $\gamma_{\mu}$ and $\gamma_{\text {nuc }}$, experimental relaxation rates $1 / T_{1}^{\exp }$ in $\mathrm{YBa}_{2} \mathrm{Cu}_{3} \mathrm{O}_{y}$ at $\sim 100 \mathrm{~K}$ (Ref. [64]), and estimated rates $1 / T_{1}^{\text {est }}$ from loop-current magnetic fluctuations.

\begin{tabular}{lccccc}
\hline \hline & $\mu^{+}$ & ${ }^{63} \mathrm{Cu}$ & ${ }^{137} \mathrm{Ba}$ & ${ }^{17} \mathrm{O}$ & ${ }^{89} \mathrm{Y}$ \\
\hline$\gamma_{\mu, \text { nuc }}\left(10^{7} \mathrm{~s}^{-1} \mathrm{~T}^{-1}\right)$ & 85.156 & 7.1088 & 2.988 & -3.6279 & -1.3155 \\
$1 / T_{1}^{\text {exp }}\left(\mathrm{s}^{-1}\right)$ & $\sim 1000$ & $\sim 2000$ & $\sim 20$ & $\sim 30$ & $\sim 0.02$ \\
$1 / T_{1}^{\text {est }}\left(\mathrm{s}^{-1}\right)$ & & 7.0 & 1.2 & 1.8 & 0.2 \\
\hline \hline
\end{tabular}

shown $[31,61]$ that these issues are not related to the observed maxima in the $\mu^{+}$relaxation rates at $T_{\mathrm{mag}} \sim T^{*}$. We note that much of the data of Ref. [31] and all of the present results were obtained for LF strong enough to decouple static nuclear dipolar relaxation [54], and they are thus not subject to a recent critique [38].

\section{Loop-current fluctuations and NMR}

From our results $\lambda\left(H_{L}=0\right)=2\left(\gamma_{\mu} B_{\mathrm{loc}}^{\mathrm{rms}}\right)^{2} \tau_{c} \approx 1 \mathrm{~ms}^{-1}$ (Figs. 3 and 4), we can roughly estimate nuclear spin-lattice (dynamic) relaxation rates $1 / T_{1}$ contributed by putative IUC loop currents $[20,21,62,63]$. Assuming fluctuating fields at nuclear sites with similar magnitudes and correlation times as at muon sites, we scale our $\mu$ SR rates by factors $\left(\gamma_{\text {nuc }} / \gamma_{\mu}\right)^{2}$, where $\gamma_{\text {nuc }}$ is the nuclear gyromagnetic ratio, to obtain estimates $1 / T_{1}^{\text {est }}$ of NMR rates. Loop currents flow between ions and are spin-free, so that spin hyperfine coupling is not involved. Intersite orbital matrix elements might contribute to NMR relaxation, but they have not been estimated [64].

Table II compares values of $1 / T_{1}^{\text {est }}$ with experimental values $1 / T_{1}^{\text {exp }}$ at $\sim 100 \mathrm{~K}$ for ${ }^{63} \mathrm{Cu},{ }^{137} \mathrm{Ba}{ }^{17} \mathrm{O}$, and ${ }^{89} \mathrm{Y}$ NMR in $\mathrm{YBa}_{2} \mathrm{Cu}_{3} \mathrm{O}_{y}$ [64]. With the exception of ${ }^{89} \mathrm{Y}$, the estimated NMR rates are more than an order of magnitude smaller than the measured rates, making observation of the loop-current contributions difficult. Furthermore, the NMR rates would be suppressed by the Redfield field dependence [Eq. (1)] for applied fields greater than a few tesla. Observation would also be difficult for ${ }^{89} \mathrm{Y} N \mathrm{NR}$; the $\mathrm{Y}$ site in the $\mathrm{YBa}_{2} \mathrm{Cu}_{3} \mathrm{O}_{y}$ crystal structure is symmetric with respect to oppositely directed IUC loop currents, and the local fields there would cancel [44]. Absent further information on an intersite orbital hyperfine contribution, the LF- $\mu$ SR results are not in conflict with the absence of NMR evidence for the slow fluctuations.

\section{Hidden-order phase of $\operatorname{Sr}_{2} \mathrm{Ir}_{1-x} \mathrm{Rh}_{x} \mathrm{O}_{4}$}

It is intriguing that similar unusual magnetic fluctuations have been observed via the Redfield field dependence of $\mu^{+}$dynamic relaxation in the "hidden-order" phase of $\mathrm{Sr}_{2} \mathrm{Ir}_{1-x} \mathrm{Rh}_{x} \mathrm{O}_{4}$ [58]. The hole-doped cuprates and the $\mathrm{Rh}$ doped iridates share similar crystal symmetry, and there is a similarity in electronic structure and magnetic order geometry as well $[18,65]$. In addition, PND experiments found evidence for TRS breaking [66]. Both $\tau_{c}$ and $B_{\mathrm{loc}}^{\text {rms }}$ are of the same magnitude as in $\mathrm{YBa}_{2} \mathrm{Cu}_{3} \mathrm{O}_{y}$, suggesting that slow spin dynamics might have the same origin in both systems.

\section{CONCLUSIONS}

Using LF- $\mu$ SR, we have measured the correlation times $\tau_{c}$ and rms dynamic local fields $B_{\mathrm{loc}}^{\mathrm{rms}}$ at muon sites associated with slow magnetic fluctuations in the pseudogap phase of $\mathrm{YBa}_{2} \mathrm{Cu}_{3} \mathrm{O}_{y}, y=6.77$ and 6.83. Our results show that the fluctuating IUD magnetism of this phase persists down to the superconducting transition. Although $\mu \mathrm{SR}$ does not yield direct information on the spatial structure of the fluctuating magnetization, the rate maxima at $T_{\mathrm{mag}} \approx T^{*}$ and the consistency of the magnitude of $B_{\mathrm{loc}}^{\mathrm{rms}}$ with the IUC moment values from PND experiments are evidence that the fluctuating fields arise from IUC moments. Persistent dynamic relaxation in the pseudogap phase has been attributed to quantum size effects in disordered loop-current domains [48]; an alternative scenario might involve a macroscopically degenerate ground state. The long but finite correlation times are perhaps conceptually similar to long-range order in the presence of long but finite correlation lengths [67]. More work is needed to understand this situation. Finally, recent reports $[68,69]$ suggest interesting behavior in overdoped cuprate samples with $p>0.19$, where the pseudogap phase is usually believed to vanish. This overdoped region should be studied further by $\mu \mathrm{SR}$.

\section{ACKNOWLEDGMENTS}

We wish to thank P. Bourges, R. Kadono, Y. Matsuda, and C. M. Varma for fruitful discussions. We are grateful to D. J. Arseneau and B. Hitti of TRIUMF, the staff of J-PARC ARTEMIS (2017B0024), and the ISIS Cryogenics Group for valuable help during the $\mu$ SR experiments. We also thank D. C. Peets for suggestions on crystal preparation. This work was funded by the National Research and Development Program of China, Grants No. 2017YFA0303104, No. 2016YFA0300503, and No. 2016YFA0300403; the National Natural Science Foundations of China, Grants No. 11774061 and No. 12034004; Shanghai Municipal Science and Technology Major Project (Grant No. 2019SHZDZX01 and No. 20ZR1405300); the US National Science Foundation, Grants No. DMR/PREM-1523588, No. HRD-1547723, and No. DMR-1905636; and the Academic Senate of the University of California, Riverside.

\section{APPENDIX A: $\mu$ SR DATA ANALYSIS, BACKGROUND CORRECTIONS}

\section{Data analysis}

LF- $\mu$ SR experiments typically involve two opposing positron counters $(+$ and -$)$, oriented parallel to the initial muon spin polarization [39-41]. Counts $N^{ \pm}(t)$ from the counters are analyzed using the corresponding two expressions

$$
N^{ \pm}(t)=N^{ \pm}(0) e^{-t / \tau_{\mu}}\left[1 \pm A^{ \pm}(t)\right],
$$

where $\tau_{\mu}=2.197 \mu \mathrm{s}$ is the muon beta-decay lifetime. The asymmetry relaxation functions $A^{ \pm}(t)$ are given by

$$
A^{ \pm}(t)=A_{0}^{ \pm} G(t),
$$

with initial values $A_{0}^{ \pm}$and a normalized relaxation function $G(t)$. The $N^{ \pm}(0)$ and $A_{0}^{ \pm}$are spectrometer-dependent.

Two approaches are commonly used to fit these expressions to the data: "separate-histogram" fits, in which Eqs. (A1) 
are fit separately with a common $G(t)$; and "asymmetry-plot" fits [70], in which parameters $\alpha=N^{-}(0) / N^{+}(0)$ and $\beta=$ $A_{0}^{-} / A_{0}^{+}$are introduced, and $N^{+}(0)$ and $\tau_{\mu}$ are eliminated from Eqs. (A1). This yields a single equation for $A_{0}^{+} G(t)$.

\section{2. $\alpha$ checks}

At each temperature, we first performed a so-called $\alpha$ check run in a weak (2-mT) transverse field, where the oscillations provide accurate determinations of $N^{ \pm}(0)$ (and thus $\alpha$ ) and $A_{0}^{ \pm}$(and thus $\beta$ ). Parameters without field dependence were fixed during the LF fits. LF values $\gtrsim 0.1 \mathrm{~T}$ influence $\alpha$, so it was left free in the fits. Separate $\alpha$ checks for each temperature determine parameter changes due to thermal expansion in the cryostat.

\section{Background correction}

There are two classes of spurious background positron counts in $\mu \mathrm{SR}$ experiments. For a continuous-beam muon source such as at TRIUMF, counts from a counter in the muon beam initiate the detection of an event. A small uncorrelated (time-independent) background is present due to muons not registered by this counter; their positrons contribute (small) constant additive terms $B^{ \pm}$to Eqs. (A1). For asymmetry-plot fits, " $t<0$ " data from times earlier than muon stops are normally used to estimate the $B^{ \pm}$. Bueno et al. [59] found, however, that this introduces a systematic error in ultraslow relaxation measurements. Separate-histogram fits, where the $B^{ \pm}$are fit parameters, were therefore used in the present study.

Correlated background is due to muons that trigger the muon counter but miss the sample and stop in the sample holder or cryostat. It is spectrometer-dependent and, as noted in Sec. II, the correction procedures for LAMPF and ARTEMIS data are different.

In the TRIUMF VG-Quant gas-flow cryostat, the amplitude of the signal from the sample support frame is small, but its relaxation rate is significant. To estimate the correction we obtained data from the frame itself with the sample removed. TRIUMF spectrometer scalers provide total counts of incoming muons $\left(T_{M}\right)$ and "gated" (nonvetoed) muons $\left(\mu_{g}\right)$, so that the fraction of gated events is $\mu_{g} / T_{M}$. These were recorded for the "empty-frame" data and separately for "total" (i.e., sample + frame) data with a pure silver sample. Then the fraction $\eta_{f}$ of the frame signal is

$$
\eta_{f}=\frac{\left(\mu_{g} / T_{M}\right)_{f}}{\left(\mu_{g} / T_{M}\right)_{\mathrm{tot}}} .
$$

This yields $\eta_{f}=0.0267$

The observed asymmetry relaxation $A^{\text {tot }}(t)$ with the sample present is then ( $s$ denotes sample, $f$ denotes frame)

$$
A^{\mathrm{tot}}(t)=A_{0}^{\mathrm{tot}} G^{\mathrm{tot}}(t)=\eta_{s} A_{0}^{s} G_{s}(t)+\eta_{f} A_{0}^{f} G_{f}(t),
$$

where $\eta_{s}=1-\eta_{f}$, the $A_{0}$ 's are initial asymmetries, and the $G(t)$ 's are relaxation functions, assumed exponential. Both relaxation rates are small, so that the signals decay approximately linearly (cf. Fig. 1):

$$
\begin{aligned}
A^{\mathrm{tot}}(t) & =A_{0}^{\mathrm{tot}}\left[1-\lambda_{\mathrm{tot}} t\right] \\
& =\eta_{s} A_{0}^{s}\left(1-\lambda_{s} t\right)+\eta_{f} A_{0}^{f}\left(1-\lambda_{f} t\right) \\
& =\left(\eta_{s} A_{0}^{s}+\eta_{f} A_{0}^{f}\right)-\left(\eta_{s} A_{0}^{s} \lambda_{s}+\eta_{f} A_{0}^{f} \lambda_{f}\right) t .
\end{aligned}
$$

Thus

$$
A_{0}^{\text {tot }}=\eta_{s} A_{0}^{s}+\eta_{f} A_{0}^{f}
$$

and

$$
\lambda_{\text {tot }}=\frac{\eta_{s} A_{0}^{s} \lambda_{s}+\eta_{f} A_{0}^{f} \lambda_{f}}{A_{0}^{\text {tot }}},
$$

so that

$$
\lambda_{s}=\frac{\lambda_{\text {tot }}-\eta_{f}\left(A_{0}^{f} / A_{0}^{\mathrm{tot}}\right) \lambda_{f}}{1-\eta_{f}\left(A_{0}^{f} / A_{0}^{\mathrm{tot}}\right)} .
$$

Since $A_{0}^{f} / A_{0}^{\text {tot }}<1$, the denominator in Eq. (A8) is $\lesssim 2 \%$ less than unity. This is well within the uncertainty in the numerator, so that the correction of the LF data can be made simply by fitting the function

$$
A^{\text {tot }}(t)=A_{0}^{\text {tot }} \exp \left[-\left(\lambda_{L}+\lambda_{\text {corr }}\right) t\right]
$$

to the data, with $\lambda_{\text {corr }}=\eta_{f}\left(A_{0}^{f} / A_{0}^{\text {tot }}\right) \lambda_{f}=0.4 \mathrm{~ms}^{-1}$.

For ARTEMIS data, the sizable signal from the Ag sample holder was taken into account in the fitting function. Its fraction $\eta_{\mathrm{Ag}}$ was obtained by fitting ZF data, where the sample and $\mathrm{Ag}$ relaxation rates are very different; this allows amplitudes of the two contributions to be determined. With $\lambda_{\mathrm{Ag}}=1 \mathrm{~ms}^{-1}$ from Fig. 2, the LF relaxation function is

$$
G(t)=\left(1-\eta_{\mathrm{Ag}}\right) \exp \left(-\lambda_{L} t\right)+\eta_{\mathrm{Ag}} \exp \left(-\lambda_{\mathrm{Ag}} t\right) .
$$

\section{Oscillating component}

The $\mu^{+}$beam spin polarization is initially (anti)parallel to the beam direction, but it precesses slightly as the beam passes through a separator (crossed electric and magnetic fields) that removes positron contamination from the muon beam. Thus there is always a small angle between the stopped $\mu^{+}$initial spin direction and the applied field (which is parallel to the beam).

The resulting $\mu^{+}$precession at frequency $\gamma_{\mu} \mu_{0} H_{L}$ would not contribute to the LF signal if the spectrometer were perfectly axially symmetric, but a small oscillating component is sometimes observed. The oscillations identify this signal, and it is easily included in the fit function if it is present [no oscillations were observed in the data of Fig. 1(a)].

\section{APPENDIX B: STATISTICAL DATA}

The uncertainties in the ultraslow relaxation rates are large, and some of the fit curves in Figs. 3 and 4 appear to be only distantly related to the data. We therefore wish to emphasize that all fits were obtained using conventional nonlinear least-squares techniques, which produce the values and uncertainties of the parameters shown in Fig. 5 in the usual way.

The parameter uncertainties in this article are standard deviations $\sigma$. The inverse relative standard deviation (IRSD) of a parameter is its value divided by its standard deviation, and it is the $N$ in " $N \sigma$ " that is commonly used to describe the significance of the results. The cumulative IRSD is the square root of the sum of squares of the individual IRSDs, and it is a measure for the entire dataset.

Reduced $\chi^{2}$ values and IRSDs of $\tau_{c}$ and $B_{\mathrm{loc}}^{\mathrm{rms}}$ from fits shown in Figs. 3 and 4 are listed in Tables III and IV, 
TABLE III. Statistical data for $\mathrm{YBa}_{2} \mathrm{Cu}_{3} \mathrm{O}_{6.77}$ : reduced $\chi^{2}$ and IRSDs of $\tau_{c}$ and $B_{\mathrm{loc}}^{\mathrm{rms}}$ from fits of the Redfield relation [Eq. (1)] to the field dependencies of the relaxation rates (Fig. 3).

\begin{tabular}{lccc}
\hline \hline & & \multicolumn{2}{c}{ IRSD } \\
\cline { 3 - 4 }$T(\mathrm{~K})$ & $\chi^{2}$ & $\tau_{c}$ & $B_{\mathrm{loc}}^{\text {rms }}$ \\
\hline 85 & 1.78 & 3.2 & 7.2 \\
95 & 0.50 & 4.8 & 11.2 \\
110 & 1.75 & 2.1 & 4.8 \\
125 & 1.02 & 2.7 & 6.1 \\
139 & 1.02 & 1.3 & 2.9 \\
157 & 0.90 & 2.6 & 6.5 \\
188 & 0.38 & 2.2 & 5.0 \\
Cumulative & & 7.63 & 17.69 \\
\hline \hline
\end{tabular}

respectively. The scatter in $\chi^{2}$ is consistent with the number of degrees of freedom in the data. Given the uncertainties, it is not surprising that many of the ISRDs are smaller than the usual standard of 5 . The cumulative values for both
TABLE IV. Statistical data for $\mathrm{YBa}_{2} \mathrm{Cu}_{3} \mathrm{O}_{6.83}$ : reduced $\chi^{2}$ and IRSDs of $\tau_{c}$ and $B_{\mathrm{loc}}^{\mathrm{rms}}$ from fits of the Redfield relation [Eq. (1)] to the field dependencies of the relaxation rates (Fig. 4).

\begin{tabular}{lccc}
\hline \hline & & \multicolumn{2}{c}{ IRSD } \\
\cline { 3 - 4 }$T(\mathrm{~K})$ & $\chi^{2}$ & $\tau_{c}$ & $B_{\text {loc }}^{\text {rms }}$ \\
\hline 93 & 0.97 & 3.6 & 8.4 \\
105 & 0.53 & 3.0 & 6.9 \\
112 & 1.17 & 2.9 & 6.7 \\
119 & 0.40 & 4.7 & 10.9 \\
126 & 1.06 & 2.9 & 6.7 \\
135 & 0.93 & 0.9 & 1.9 \\
145 & 0.97 & 2.0 & 4.6 \\
155 & 0.30 & 3.9 & 9.5 \\
170 & $\mathrm{~N} / \mathrm{A}^{\mathrm{a}}$ & & \\
Cumulative & & 8.99 & 21.01 \\
\hline \hline
\end{tabular}

${ }^{\mathrm{a} D a t a}$ not fit; cf. Sec. III B.

samples are, however, quite significant, and they are evidence for nonzero $\tau_{c}$ and $B_{\mathrm{loc}}^{\mathrm{rms}}$.
[1] V. J. Emery and S. A. Kivelson, Importance of phase fluctuations in superconductors with small superfluid density, Nature (London) 374, 434 (1995).

[2] T. Timusk and B. Statt, The pseudogap in high-temperature superconductors: an experimental survey, Rep. Prog. Phys. 62, 61 (1999).

[3] P. A. Lee, Pseudogaps in underdoped cuprates, Physica C 317318, 194 (1999).

[4] S. A. Kivelson, I. P. Bindloss, E. Fradkin, V. Oganesyan, J. M. Tranquada, A. Kapitulnik, and C. Howald, How to detect fluctuating stripes in the high-temperature superconductors, Rev. Mod. Phys. 75, 1201 (2003).

[5] M. R. Norman and C. Pépin, The electronic nature of high temperature cuprate superconductors, Rep. Prog. Phys. 66, 1547 (2003).

[6] D. N. Basov and T. Timusk, Electrodynamics of high- $T_{c}$ superconductors, Rev. Mod. Phys. 77, 721 (2005).

[7] M. R. Norman, D. Pines, and C. Kallin, The pseudogap: friend or foe of high $T_{c}$ ? Adv. Phys. 54, 715 (2005).

[8] C. M. Varma, Mind the pseudogap, Nature (London) 468, 184 (2010).

[9] M. R. Norman, The challenge of unconventional superconductivity, Science 332, 196 (2011).

[10] B. Keimer, S. A. Kivelson, M. R. Norman, S. Uchida, and J. Zaanen, From quantum matter to high-temperature superconductivity in copper oxides, Nature (London) 518, 179 (2015).

[11] C. Proust and L. Taillefer, The remarkable underlying ground states of cuprate superconductors, Annu. Rev. Condens. Matter Phys. 10, 409 (2019).

[12] J. Loram, K. Mirza, J. Wade, J. Cooper, and W. Liang, The electronic specific heat of cuprate superconductors, Physica C 235-240, 134 (1994).

[13] B. Fauqué, Y. Sidis, V. Hinkov, S. Pailhès, C. T. Lin, X. Chaud, and P. Bourges, Magnetic Order in the Pseudogap Phase of High- $T_{C}$ Superconductors, Phys. Rev. Lett. 96, 197001 (2006).
[14] Y. Li, V. Balédent, N. Barišić, Y. Cho, B. Fauqué, Y. Sidis, G. Yu, X. Zhao, P. Bourges, and M. Greven, Unusual magnetic order in the pseudogap region of the superconductor $\mathrm{HgBa}_{2} \mathrm{CuO}_{4+\delta}$, Nature (London) 455, 372 (2008).

[15] J. Xia, E. Schemm, G. Deutscher, S. A. Kivelson, D. A. Bonn, W. N. Hardy, R. Liang, W. Siemons, G. Koster, M. M. Fejer, and A. Kapitulnik, Polar Kerr-Effect Measurements of the High-Temperature $\mathrm{YBa}_{2} \mathrm{Cu}_{3} \mathrm{O}_{6+x}$ Superconductor: Evidence for Broken Symmetry Near the Pseudogap Temperature, Phys. Rev. Lett. 100, 127002 (2008).

[16] L. Mangin-Thro, Y. Sidis, A. Wildes, and P. Bourges, Intra-unit-cell magnetic correlations near optimal doping in $\mathrm{YBa}_{2} \mathrm{Cu}_{3} \mathrm{O}_{6.85}$, Nat. Commun. 6, 7705 (2015).

[17] Y. Lubashevsky, L. D. Pan, T. Kirzhner, G. Koren, and N. P. Armitage, Optical Birefringence and Dichroism of Cuprate Superconductors in the THz Regime, Phys. Rev. Lett. 112, 147001 (2014).

[18] L. Zhao, C. A. Belvin, R. Liang, D. A. Bonn, W. N. Hardy, N. P. Armitage, and D. Hsieh, A global inversion-symmetry-broken phase inside the pseudogap region of $\mathrm{YBa}_{2} \mathrm{Cu}_{3} \mathrm{O}_{y}$, Nat. Phys. 13, 250 (2016).

[19] Y. Sato, S. Kasahara, H. Murayama, Y. Kasahara, E.-G. Moon, T. Nishizaki, T. Loew, J. Porras, B. Keimer, T. Shibauchi, and Y. Matsuda, Thermodynamic evidence for a nematic phase transition at the onset of the pseudogap in $\mathrm{YBa}_{2} \mathrm{Cu}_{3} \mathrm{O}_{y}$, Nat. Phys. 13, 1074 (2017).

[20] C. M. Varma, Non-Fermi-liquid states and pairing instability of a general model of copper oxide metals, Phys. Rev. B 55, 14554 (1997).

[21] M. E. Simon and C. M. Varma, Detection and Implications of a Time-Reversal Breaking State in Underdoped Cuprates, Phys. Rev. Lett. 89, 247003 (2002).

[22] A. S. Moskvin, Pseudogap phase in cuprates: Oxygen orbital moments instead of circulating currents, JETP Lett. 96, 385 (2012). 
[23] M. Fechner, M. J. A. Fierz, F. Thöle, U. Staub, and N. A. Spaldin, Quasistatic magnetoelectric multipoles as order parameter for pseudogap phase in cuprate superconductors, Phys. Rev. B 93, 174419 (2016).

[24] P. Bourges, D. Bounoua, and Y. Sidis, Loop currents in quantum matter, arXiv:2103.13295.

[25] T. P. Croft, E. Blackburn, J. Kulda, R. Liang, D. A. Bonn, W. N. Hardy, and S. M. Hayden, No evidence for orbital loop currents in charge-ordered $\mathrm{YBa}_{2} \mathrm{Cu}_{3} \mathrm{O}_{6+x}$ from polarized neutron diffraction, Phys. Rev. B 96, 214504 (2017).

[26] T. P. Croft, E. Blackburn, J. Kulda, R. Liang, D. A. Bonn, W. N. Hardy, and S. M. Hayden, Reply to "Comment on 'No evidence for orbital loop currents in charge-ordered $\mathrm{YBa}_{2} \mathrm{Cu}_{3} \mathrm{O}_{6+x}$ from polarized neutron diffraction', ” Phys. Rev. B 98, 016502 (2018).

[27] P. Bourges, Y. Sidis, and L. Mangin-Thro, Comment on "No evidence for orbital loop currents in charge-ordered $\mathrm{YBa}_{2} \mathrm{Cu}_{3} \mathrm{O}_{6+x}$ from polarized neutron diffraction," Phys. Rev. B 98, 016501 (2018)

[28] P. Bourges, D. Bounoua, J. Jeong, L. Mangin-Thro, and Y. Sidis, Evidence for intra-unit cell magnetism in superconducting cuprates: a technical assessment, J. Phys.: Conf. Ser. 1316, 012003 (2019).

[29] See, for example, M. Minola, G. Dellea, H. Gretarsson, Y. Y. Peng, Y. Lu, J. Porras, T. Loew, F. Yakhou, N. B. Brookes, Y. B. Huang, J. Pelliciari, T. Schmitt, G. Ghiringhelli, B. Keimer, L. Braicovich, and M. Le Tacon, Collective Nature of Spin Excitations in Superconducting Cuprates Probed by Resonant Inelastic X-Ray Scattering, Phys. Rev. Lett. 114, 217003 (2015).

[30] J. Sonier, J. Brewer, R. Kiefl, R. Miller, G. Morris, C. Stronach, J. Gardner, S. Dunsiger, D. Bonn, W. Hardy et al., Anomalous weak magnetism in superconducting $\mathrm{YBa}_{2} \mathrm{Cu}_{3} \mathrm{O}_{6+x}$, Science 292, 1692 (2001).

[31] J. Zhang, Z. Ding, C. Tan, K. Huang, O. O. Bernal, P.-C. Ho, G. D. Morris, A. D. Hillier, P. K. Biswas, S. P. Cottrell, H. Xiang, X. Yao, D. E. MacLaughlin, and L. Shu, Discovery of slow magnetic fluctuations and critical slowing down in the pseudogap phase of $\mathrm{YBa}_{2} \mathrm{Cu}_{3} \mathrm{O}_{y}$, Sci. Adv. 4, eaao5235 (2018).

[32] A. Pal, S. R. Dunsiger, K. Akintola, A. C. Y. Fang, A. Elhosary, M. Ishikado, H. Eisaki, and J. E. Sonier, Quasistatic internal magnetic field detected in the pseudogap phase of $\mathrm{Bi}_{2+x} \mathrm{Sr}_{2-x} \mathrm{CaCu}_{2} \mathrm{O}_{8+\delta}$ by muon spin relaxation, Phys. Rev. B 97, 060502(R) (2018).

[33] J. E. Sonier, J. H. Brewer, R. F. Kiefl, R. H. Heffner, K. F. Poon, S. L. Stubbs, G. D. Morris, R. I. Miller, W. N. Hardy, R. Liang, D. A. Bonn, J. S. Gardner, C. E. Stronach, and N. J. Curro, Correlations between charge ordering and local magnetic fields in overdoped $\mathrm{YBa}_{2} \mathrm{Cu}_{3} \mathrm{O}_{6+x}$, Phys. Rev. B 66, 134501 (2002).

[34] G. J. MacDougall, A. A. Aczel, J. P. Carlo, T. Ito, J. Rodriguez, P. L. Russo, Y. J. Uemura, S. Wakimoto, and G. M. Luke, Absence of Broken Time-Reversal Symmetry in the Pseudogap State of the High-Temperature $\mathrm{La}_{2-x} \mathrm{Sr}_{x} \mathrm{CuO}_{4}$ Superconductor from Muon-Spin-Relaxation Measurements, Phys. Rev. Lett. 101, 017001 (2008).

[35] J. E. Sonier, V. Pacradouni, S. A. Sabok-Sayr, W. N. Hardy, D. A. Bonn, R. Liang, and H. A. Mook, Detection of the Unusual Magnetic Orders in the Pseudogap Region of a High-Temperature Superconducting $\mathrm{YBa}_{2} \mathrm{Cu}_{3} \mathrm{O}_{6.6}$ Crystal by Muon-Spin Relaxation, Phys. Rev. Lett. 103, 167002 (2009).
[36] W. Huang, V. Pacradouni, M. P. Kennett, S. Komiya, and J. E. Sonier, Precision search for magnetic order in the pseudogap regime of $\mathrm{La}_{2-x} \mathrm{Sr}_{x} \mathrm{CuO}_{4}$ by muon spin relaxation, Phys. Rev. B 85, 104527 (2012).

[37] A. Pal, K. Akintola, M. Potma, M. Ishikado, H. Eisaki, W. N. Hardy, D. A. Bonn, R. Liang, and J. E. Sonier, Investigation of potential fluctuating intra-unit cell magnetic order in cuprates by $\mu$ SR, Phys. Rev. B 94, 134514 (2016).

[38] S. Gheidi, K. Akintola, A. C. Y. Fang, S. Sundar, A. M. Côté, S. R. Dunsiger, G. D. Gu, and J. E. Sonier, Absence of $\mu \mathrm{SR}$ evidence for magnetic order in the pseudogap phase of $\mathrm{Bi}_{2+x} \mathrm{Sr}_{2-x} \mathrm{CaCu}_{2} \mathrm{O}_{8+\delta}$, Phys. Rev. B 101, 184511 (2020).

[39] S. J. Blundell, Spin-polarized muons in condensed matter physics, Contemp. Phys. 40, 175 (1999).

[40] J. H. Brewer, Muon spin rotation/relaxation/resonance, in Digital Encyclopedia of Applied Physics, edited by G. L. Trigg, E. S. Vera, and W. Greulich (Wiley-VCH Verlag, Weinheim, 2003).

[41] A. Yaouanc and P. Dalmas de Réotier, Muon Spin Rotation, Relaxation, and Resonance: Applications to Condensed Matter, International Series of Monographs on Physics (Oxford University Press, New York, 2011).

[42] S. Strässle, J. Roos, M. Mali, H. Keller, and T. Ohno, Lack of Evidence for Orbital-Current Effects in the High-Temperature $\mathbf{Y}_{2} \mathrm{Ba}_{4} \mathrm{Cu}_{7} \mathbf{O}_{15-\delta}$ Superconductor Using ${ }^{89} \mathrm{Y}$ Nuclear Magnetic Resonance, Phys. Rev. Lett. 101, 237001 (2008).

[43] S. Strässle, B. Graneli, M. Mali, J. Roos, and H. Keller, Absence of Orbital Currents in Superconducting $\mathrm{YBa}_{2} \mathrm{Cu}_{4} \mathrm{O}_{8}$ Using a Zeeman-Perturbed Nuclear-Quadrupole-Resonance Technique, Phys. Rev. Lett. 106, 097003 (2011).

[44] A. M. Mounce, S. Oh, J. A. Lee, W. P. Halperin, A. P. Reyes, P. L. Kuhns, M. K. Chan, C. Dorow, L. Ji, D. Xia, X. Zhao, and M. Greven, Absence of Static Loop-Current Magnetism at the Apical Oxygen Site in $\mathrm{HgBa}_{2} \mathrm{CuO}_{4+\delta}$ from NMR, Phys. Rev. Lett. 111, 187003 (2013).

[45] J. E. Sonier, Comment on "Discovery of slow magnetic fluctuations and critical slowing down in the pseudogap phase of $\mathrm{YBa}_{2} \mathrm{Cu}_{3} \mathrm{O}_{y}$, , arXiv:1706.03023.

[46] T. Wu, H. Mayaffre, S. Krämer, M. Horvatić, C. Berthier, W. N. Hardy, R. X. Liang, D. A. Bonn, and M.-H. Julien, Incipient charge order observed by NMR in the normal state of $\mathrm{YBa}_{2} \mathrm{Cu}_{3} \mathrm{O}_{y}$, Nat. Commun. 6, 6438 (2015).

[47] See, for example, Ref. [41], Chap. 10.

[48] C. M. Varma, Pseudogap in cuprates in the loop-current ordered state, J. Phys.: Condens. Matter 26, 505701 (2014).

[49] H. Xiang, L. Guo, H. Li, X. Cui, J. Qian, G. Hussain, Y. Liu, X. Yao, Q. Rao, and Z. Zou, Polythermal method of top-seeded solution-growth for large-sized single crystals of $\mathrm{REBa}_{2} \mathrm{Cu}_{3} \mathrm{O}_{7-\delta}$, Scr. Mater. 116, 36 (2016).

[50] Y. Shiohara and A. Endo, Crystal growth of bulk high- $T_{c}$ superconducting oxide materials, Mater. Sci. Eng., R 19, 1 (1997).

[51] P. Schleger, W. Hardy, and B. Yang, Thermodynamics of oxygen in $\mathrm{Y}_{1} \mathrm{Ba}_{2} \mathrm{Cu}_{3} \mathrm{O}_{x}$ between $450^{\circ} \mathrm{C}$ and $650^{\circ} \mathrm{C}$, Physica $\mathrm{C} 176$, 261 (1991).

[52] H. Gao, C. Ren, L. Shan, Y. Wang, Y. Zhang, S. Zhao, X. Yao, and H.-H. Wen, Reversible magnetization and critical fluctuations in systematically doped $\mathrm{YBa}_{2} \mathrm{Cu}_{3} \mathrm{O}_{7-\delta}$ single crystals, Phys. Rev. B 74, 020505(R) (2006).

[53] A. Suter and B. Wojek, Musrfit: A free platform-independent framework for $\mu$ SR data analysis, Phys. Proc. 30, 69 (2012), 
12th International Conference on Muon Spin Rotation, Relaxation and Resonance ( $\mu$ SR2011).

[54] R. S. Hayano, Y. J. Uemura, J. Imazato, N. Nishida, T. Yamazaki, and R. Kubo, Zero- and low-field spin relaxation studied by positive muons, Phys. Rev. B 20, 850 (1979).

[55] C. P. Slichter, Principles of Magnetic Resonance, 3rd ed., Springer Series in Solid-State Sciences (Springer-Verlag, New York, 1996).

[56] Y. Aoki, A. Tsuchiya, T. Kanayama, S. R. Saha, H. Sugawara, H. Sato, W. Higemoto, A. Koda, K. Ohishi, K. Nishiyama, and R. Kadono, Time-Reversal Symmetry-Breaking Superconductivity in Heavy-Fermion $\mathrm{PrOs}_{4} \mathrm{Sb}_{12}$ Detected by Muon-Spin Relaxation, Phys. Rev. Lett. 91, 067003 (2003).

[57] Y. Li, D. Adroja, P. K. Biswas, P. J. Baker, Q. Zhang, J. Liu, A. A. Tsirlin, P. Gegenwart, and Q. Zhang, Muon Spin Relaxation Evidence for the U(1) Quantum Spin-Liquid Ground State in the Triangular Antiferromagnet $\mathrm{YbMgGaO}_{4}$, Phys. Rev. Lett. 117, 097201 (2016).

[58] C. Tan, Z. F. Ding, J. Zhang, Z. H. Zhu, O. O. Bernal, P. C. Ho, A. D. Hillier, A. Koda, H. Luetkens, G. D. Morris, D. E. MacLaughlin, and L. Shu, Slow magnetic fluctuations and critical slowing down in $\mathrm{Sr}_{2} \mathrm{Ir}_{1-x} \mathrm{Rh}_{x} \mathrm{O}_{4}$, Phys. Rev. B 101, 195108 (2020).

[59] J. F. Bueno, D. J. Arseneau, R. Bayes, J. H. Brewer, W. Faszer, M. D. Hasinoff, G. M. Marshall, E. L. Mathie, R. E. Mischke, G. D. Morris, K. Olchanski, V. Selivanov, and R. Tacik, Longitudinal muon spin relaxation in high-purity aluminum and silver, Phys. Rev. B 83, 205121 (2011).

[60] M. S. Grønsleth, T. B. Nilssen, E. K. Dahl, E. B. Stiansen, C. M. Varma, and A. Sudbø, Thermodynamic properties near the onset of loop-current order in high- $T_{c}$ superconducting cuprates, Phys. Rev. B 79, 094506 (2009).
[61] J. Zhang, Z. F. Ding, C. Tan, K. Huang, O. O. Bernal, P.-C. Ho, G. D. Morris, A. D. Hillier, P. K. Biswas, S. P. Cottrell, H. Xiang, X. Yao, D. E. MacLaughlin, and L. Shu, Reply to "Comment on 'Discovery of slow magnetic fluctuations and critical slowing down in the pseudogap phase of $\mathrm{YBa}_{2} \mathrm{Cu}_{3} \mathrm{O}_{y}$,', arXiv: 1707.00069 .

[62] C. M. Varma, Theory of the pseudogap state of the cuprates, Phys. Rev. B 73, 155113 (2006).

[63] S. Lederer and S. A. Kivelson, Observable NMR signal from circulating current order in YBCO, Phys. Rev. B 85, 155130 (2012).

[64] R. E. Walstedt, The NMR Probe of High $T_{c}$ Materials and Correlated Electron Systems, Springer Tracts in Modern Physics Vol. 276 (Springer, Berlin, 2018).

[65] Y. K. Kim, O. Krupin, J. D. Denlinger, A. Bostwick, E. Rotenberg, Q. Zhao, J. F. Mitchell, J. W. Allen, and B. J. Kim, Fermi arcs in a doped pseudospin-1/2 Heisenberg antiferromagnet, Science 345, 187 (2014).

[66] J. Jeong, Y. Sidis, A. Louat, V. Brouet, and P. Bourges, Timereversal symmetry breaking hidden order in $\mathrm{Sr}_{2}(\mathrm{Ir}, \mathrm{Rh}) \mathrm{O}_{4}$, Nat. Commun. 8, 15119 (2017).

[67] C. M. Varma (private communication).

[68] S.-D. Chen, M. Hashimoto, Y. He, D. Song, K.-J. Xu, J.-F. He, T. P. Devereaux, H. Eisaki, D.-H. Lu, J. Zaanen, and Z.-X. Shen, Incoherent strange metal sharply bounded by a critical doping in Bi2212, Science 366, 1099 (2019).

[69] J. L. Tallon, J. G. Storey, J. R. Cooper, and J. W. Loram, Locating the pseudogap closing point in cuprate superconductors: Absence of entrant or reentrant behavior, Phys. Rev. B 101, 174512 (2020).

[70] T. M. Riseman, J. H. Brewer, and D. J. Arseneau, Corrected asymmetry plots, Hyperfine Interact. 87, 1135 (1994). 